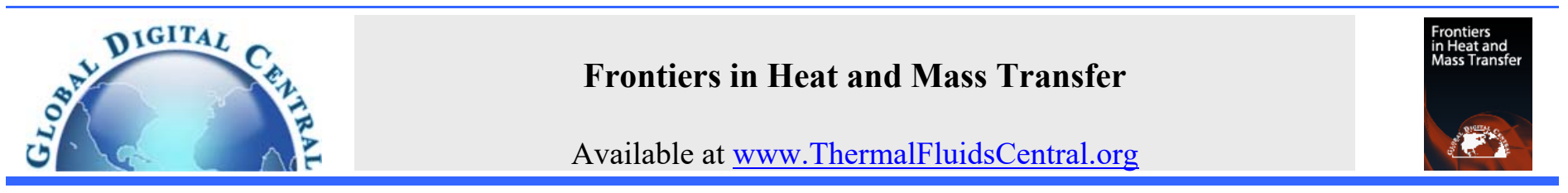

\title{
A TREE-TYPE CYLINDRICAL-SHAPED NANOPOROUS FILTERING MEMBRANE
}

\author{
Yongbin Zhang* \\ College of Mechanical Engineering, Changzhou University, Changzhou, 213164, Jiangsu Province, China
}

\begin{abstract}
A tree-type cylindrical-shaped nanoporous filtering membrane is proposed. Across the thickness of this membrane are manufactured two kinds of pores i.e. one trunk pore and four uniform branch pores, these two kinds of pores have the same homogeneous surface property and are linked together, and they are uniformly distributed on the membrane surface; The branch pore is for filtration and its radius is on the $1 \mathrm{~nm}$ or $10 \mathrm{~nm}$ scales, while the trunk pore is for collecting the flow coming from its four branch pores and it is aimed for reducing the flow resistance and increasing the flux of the membrane; The radius of the trunk pore is determined according to the radius of its branch pore. An analysis is presented for calculating the flow resistance of this membrane. It was found that the membrane thickness, the radius of the branch pore and the filtered liquid-pore interaction strength have great influences on the flow resistance of the membrane. It was also found that this membrane can be used for a liquid-liquid separation when one liquid has a weak interaction with the filtering pore, while the other liquids in the mixture have strong interactions with the filtering pore. It is evaluated that the proposed membrane has good performance characteristics including the ultimate filtration capability, the flux rate and the mechanical strength.
\end{abstract}

Keywords: Membrane; Nanopore; Filtration; Interaction; Separation

\section{INTRODUCTION}

Nanoporous filtering membranes have been in fast progress because of their capability of ultimate filtration and their application values in fine purification of substance, medicine and biology et al. (Adiga et al., 2009; Biffinger et al., 2007; Fissel et al., 2009). The filtration of these membranes relies on their nanoscale filtration pores which can finely select out the substance, however also heavily impedes the flow of liquids through the membrane. This is due to the very small pore size, the significantly increased effective liquid viscosity and the significant non-continuum effect of the liquid in the confining pore each of which can greatly reduce the flow rate through the nanopore (Zhang, 2016). People have been thrusting efforts in developing this membrane with good performances such as ultimate filtration capability, high flux rate and good mechanical strength (Li et al., 2004; Surwade et al., 2015; Venkatesan et al., 2009; Yang et al., 2006).

The author developed a new nanoporous filtration membrane across the thickness of which are manufactured two concentric cylindrical pores with different radii (Zhang, 2017a). The smaller pore is for filtration with a nanoscale radius, and the larger pore is for reducing the flow resistance and increasing the flux of the membrane. It was suggested that the depth of the smaller pore should be as small as possible and across the remaining thickness of the membrane should be manufactured the larger pore; By this way, the membrane can achieve an overall good performance characteristics. It was also shown that this membrane can be used for a liquid-liquid separation if the mixed liquids have largely different interactions with the pore walls (Zhang, 2017b).

The author has ever proposed a nanotube tree for liquid transportation which resembles a live wood tree (Zhang, 2017c). This artificial tree has a main trunk and a lot of uniform branches. It was shown that although the flow within each branch nanotube on the primitive level of the tree may be very small, the flow rate through the main trunk of the tree can be great. These functions indeed fit the requirements of ultimate filtration membranes.

Based on the earlier developed nanotube tree (Zhang, 2017c), the present paper proposes a tree-type cylindrical-shaped nanoporous filtering membrane across the thickness of which are respectively manufactured one trunk pore and its four uniform branch pores. The branch pore is for filtration with a nanoscale radius and a small depth, while the trunk pore is for reducing the flow resistance of the membrane and occupies the remaining thickness of the membrane. The radius of the trunk pore is determined according to the radius of the branch pore. An analysis is presented for calculating the flow resistance of the membrane. The performances of this membrane are evaluated as dependent on the membrane thickness, the radius of the filtration pore and the interaction between the filtered liquid and the pore walls. The capability of this membrane for a liquid-liquid separation is also investigated.

\section{MEMBRANE PICTURES}

Figures 1(a) and (b) shows the invented membrane. The uniform trunk pores with the radius $R_{b, 2}$ and the depth $l_{2}$ are evenly distributed on the membrane surface. Each trunk pore has four uniform branch pores respectively with the radius $R_{b, 1}$ and the depth $l_{1}$. The wall surfaces of the trunk pore and its branch pores have the same physical properties. The angle between the axis of the trunk pore and the axis of the branch pore is $\theta$. As Fig. 1(b) shows, the four branch pores of each trunk pore are evenly distributed on the circle with the radius $R_{d}$ on the membrane surface; On this circle, the circumferential distance between the neighboring branch pores is $\Delta_{1}$. For a dense distribution of the branch 
pores, it is normally designed that $R_{d} / R_{b, 1}=\left(4+2 k_{s}\right) / \pi$ and $k_{s}=$ $\Delta_{1} / R_{b, 1}=0.05 \sim 0.5$. The depth $l_{1}$ of the branch pore is determined according to the requirement of the mechanical strength of the filtration pore. The depth $l_{2}$ of the trunk pore is determined according to the requirement of the mechanical strength of the whole membrane. The thickness of the membrane is: $l=l_{2}+l_{1} \cos \theta$.

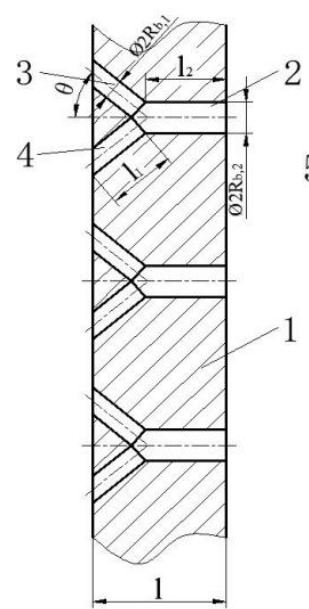

(a) Front view

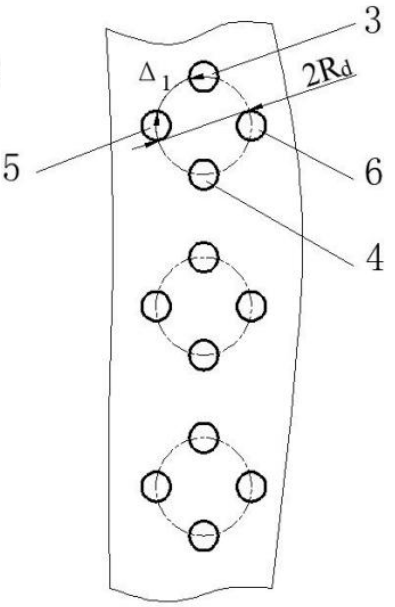

(b) Left-side view

1-Membrane substrate, 2-Trunk pore, 3, 4, 5, 6-Branch pores

Fig. 1 The studied tree-type cylindrical-shaped nanoporous filtering membrane.

\section{ANALYSIS}

The flow of the liquid in the nanoscale pores within the membrane in Figs.1(a) and (b) was simulated by using the flow factor approach model (Zhang, 2016), which has been well validated by comparison with molecular dynamics simulation results. For simplicity, the slippage of the filtered liquid on the pore wall was neglected. This could not alter the derived conclusions. According to the design principle of the nanotube tree presented by Zhang $(2017 \mathrm{c})$, the trunk pore is used to collect the flow out of its branch pores, and the radius $R_{b, 2}$ of the trunk pore is thus solved from the following equation:

$\frac{4 C q\left(\bar{R}_{b, 1}\right)\left|S\left(\bar{R}_{b, 1}\right)\right| \bar{R}_{b, 1}^{4}}{C y\left(\bar{R}_{b, 1}\right)}-\frac{C q\left(\bar{R}_{b, 2}\right)\left|S\left(\bar{R}_{b, 2}\right)\right| \bar{R}_{b, 2}^{4}}{C y\left(\bar{R}_{b, 2}\right)}=0$

where $\bar{R}_{b, 1}=R_{b, 1} / R_{c r}, \bar{R}_{b, 2}=R_{b, 2} / R_{c r}, R_{c r}$ is the critical radius of the pore for the filtered liquid to become continuum across the pore radius, $\quad C y(\bar{R})=\eta_{b f}^{\text {eff }}(\bar{R}) / \eta, C q(\bar{R})=\rho_{b f}^{\text {eff }}(\bar{R}) / \rho, \quad \rho_{b f}^{\text {eff }}$ and $\eta_{b f}^{\text {eff }}$ are respectively the average density and the effective viscosity of the filtered liquid across the pore radius, $S$ is the parameter describing the non-continuum effect of the filtered liquid across the pore radius $(-1 \leq S<0)$, and $\rho$ and $\eta$ are respectively the bulk density and the bulk viscosity of the filtered liquid at the environmental temperature and pressure.

According to the principle of the nanotube tree presented by Zhang $(2017 \mathrm{c})$, in the present membrane, the four branch pores in each pore tree is equivalent to the straight cylindrical pore with the radius $R_{b, 2}$ and with the depth $l_{1}$, and each pore tree (with one trunk pore and four branch pores) is thus equivalent to the straight cylindrical tube with the radius $R_{b, 2}$ and with the axial length $\left(l_{1}+l_{2}\right)$. If the flow resistance of this equivalent cylindrical tube is defined as $i_{f, 0}=\Delta p / q_{m, 0}$, where $\Delta p$ is the pressure drop along this tube and $q_{m, 0}$ is the mass flow rate through this tube, the flow resistance of this equivalent cylindrical tube is (Zhang, 2017d):

$i_{f, 0}=\frac{4 \eta_{b f}^{e f f}\left(\bar{R}_{b, 2}\right)\left(l_{1}+l_{2}\right)}{\pi \rho_{b f}^{e f f}\left(\bar{R}_{b, 2}\right)\left|S\left(\bar{R}_{b, 2}\right)\right| R_{b, 2}^{4}}$
The total number of the pore trees within the membrane is:

$N_{t}=\frac{A_{m} \chi}{\pi\left(l_{1} \sin \theta\right)^{2}}$

where $A_{m}$ is the area of the membrane surface and $\chi$ is the production rate of the pore tree on the membrane surface. If the flow resistance of the whole membrane is defined as $i_{f}=\Delta p / q_{m}$, where $\Delta p$ is the pressure drop on the membrane and $q_{m}$ is the total mass flow rate through the membrane, $i_{f}$ is equated as:

$i_{f}=\frac{i_{f, 0}}{N_{t}}=\frac{4 \eta_{b f}^{e f f}\left(\bar{R}_{b, 2}\right)\left(l_{1}+l_{2}\right)\left(l_{1} \sin \theta\right)^{2}}{A_{m} \chi \rho_{b f}^{e f f}\left(\bar{R}_{b, 2}\right)\left|S\left(\bar{R}_{b, 2}\right)\right| \bar{R}_{b, 2}^{4}}$

Define $I_{f}=\pi \rho R_{r}^{3} i_{f} /(4 \eta)$, where $R_{r}$ is a constant reference radius, the dimensionless flow resistance of the membrane is then written as:

$I_{f}=F\left(\bar{R}_{b, 1}\right) \frac{\pi R_{r}^{2}}{\chi A_{m}}$

Where

$F\left(\bar{R}_{b, 1}\right)=\frac{C y\left(\bar{R}_{b, 2}\right) \frac{l}{R_{b, 2}}\left(\frac{R_{d}}{l}\right)^{2}\left(\frac{l}{R_{r}}\right)^{2}}{C q\left(\bar{R}_{b, 2}\right)\left|S\left(\bar{R}_{b, 2}\right)\right|\left(\frac{R_{b, 2}}{R_{r}}\right)^{3}}\left[\sqrt{\left(1-\lambda_{2}\right)^{2}+\left(\frac{R_{d}}{l}\right)^{2}}+\lambda_{2}\right]$

Here, $\lambda_{2}=l_{2} / l$. For given values of $\chi$ and $A_{m}$, the parameter $F$ can measure the dimensionless flow resistance of the membrane. Although the value of $F$ is increased with the increase of $\lambda_{2}$, the parameter $\lambda_{2}$ has a weak influence on the value of $F$. For a better mechanical strength of the membrane and an easier manufacturing of the membrane, $\lambda_{2}$ should be close to 1.0 and $l_{1}$ should be as small as possible.

\section{EXEMPLARY CALCULATIONS}

Exemplary calculations of the values of $F$ were made for varying operational parameter values. These calculations were based on the following input parameter values: $k_{s}=0.15, \lambda_{2}=0.95$ and $R_{r}=$ $10 \mathrm{~nm}$. Weak, medium-level and strong interactions between the filtered liquid and the pore wall were respectively taken. For different liquidpore wall interactions, the average density $\rho_{b f}^{\text {eff }}$ and the effective viscosity $\eta_{b f}^{e f f}$ across the pore radius of the confined liquid and the value of the parameter $S$ describing the liquid non-continuum effect are different when the pore radius is lower than the critical radius $R_{c r}$. Stronger the liquid-pore wall interaction, larger values of $\rho_{b f}^{\text {eff }}$ and $\eta_{b f}^{e f f}$, and lower magnitudes of the parameter $S(-1 \leq S<0)$.

For whichever liquid-pore wall interaction, $C q(\bar{R})$ is generally expressed as (Zhang, 2017c and d):

$C q(\bar{R})=\left\{\begin{array}{cr}1, & \text { for } \bar{R} \geq 1 \\ m_{0}+m_{1} \bar{R}+m_{2} \bar{R}^{2}+m_{3} \bar{R}^{3}, & \text { for } 0<\bar{R}<1\end{array}\right.$

where $\bar{R}$ is $\bar{R}_{b, 1}$ or $\bar{R}_{b, 2}$ (same in the following equations), $m_{0}, m_{1}$, $m_{2}$ and $m_{3}$ are respectively constants.

Cy $(\bar{R})$ is generallyexpressed as (Zhang, 2017c and d):

$\operatorname{Cy}(\bar{R})= \begin{cases}1, & \text { for } \bar{R} \geq 1 \\ a_{0}+\frac{a_{1}}{\bar{R}}+\frac{a_{2}}{\bar{R}^{2}}, & \text { for } 0<\bar{R}<1\end{cases}$

where $a_{0}, a_{1}$ and $a_{2}$ are respectively constants.

$\mathrm{S}(\bar{R})$ is generallyexpressed as (Zhang, 2017c and d): 


$$
S(\bar{R})= \begin{cases}-1, & \text { for } \bar{R} \geq 1 \\ {\left[n_{0}+n_{1}\left(\bar{R}-n_{3}\right)^{n_{2}}\right]^{-1},} & \text { for } 0<\bar{R}<1\end{cases}
$$

where $n_{0}, n_{1}, n_{2}$ and $n_{3}$ are respectively constants.

For weak, medium-level and strong filtered liquid-pore wall interactions, the values of $R_{c r}$ were respectively taken as $3.5 \mathrm{~nm}, 10 \mathrm{~nm}$ and 20nm (Zhang, 2017c and d). For different types of the filtered liquidpore wall interaction, the values of the other parameters are respectively shown in Tables $1(\mathrm{a}-\mathrm{c})$. The values of $C q, C y$ and $S$ for different pore radii, based on the parameter values in Tables 1(a)-(c) were compared by Zhang (2014), respectively showing the weak, medium-level and strong liquid-pore wall interactions.

Table 1(a) Liquid viscosity data for different liquid-pore wall interaction types (Zhang, 2017c and d)

\begin{tabular}{|l|c|c|c|}
\hline Interaction Parameter & $a_{0}$ & $a_{1}$ & $a_{2}$ \\
\hline Strong & 1.8335 & -1.4252 & 0.5917 \\
\hline Medium & 1.0822 & -0.1758 & 0.0936 \\
\hline Weak & 0.9507 & 0.0492 & $1.6447 \mathrm{E}-4$ \\
\hline
\end{tabular}

Table 1(b) Liquid density data for different liquid-pore wall interaction types (Zhang, 2017c and d)

\begin{tabular}{|l|c|c|c|c|}
\hline Interaction Parameter & $m_{0}$ & $m_{1}$ & $m_{2}$ & $m_{3}$ \\
\hline Strong & 1.43 & -1.723 & 2.641 & -1.347 \\
\hline Medium & 1.30 & -1.065 & 1.336 & -0.571 \\
\hline Weak & 1.116 & -0.328 & 0.253 & -0.041 \\
\hline
\end{tabular}

Table 1(c) Liquid non-continuum property data for different liquid-pore wall interaction types (Zhang, 2017c and d)

\begin{tabular}{|l|c|c|c|c|}
\hline Interaction & $n_{0}$ & $n_{1}$ & $n_{2}$ & $n_{3}$ \\
\hline Strong & 0.4 & -1.374 & -0.534 & 0.035 \\
\hline Medium & -0.649 & -0.343 & -0.665 & 0.035 \\
\hline Weak & -0.1 & -0.892 & -0.084 & 0.1 \\
\hline
\end{tabular}

The calculations were also carried out for investigating the possibility of the membrane used for filtering one liquid out of other liquids (i.e. for a liquid-liquid separation). In these calculations, the flow resistances of the membrane i.e. the values of $F$ for the three liquids were respectively calculated when these liquids i.e. Liquid $\mathrm{A}$, Liquid $\mathrm{B}$ and Liquid C respectively have weak, medium-level and strong interactions with the pore wall. In these calculations, the values of the parameters for formulating $\operatorname{Cy}(\bar{R}), C q(\bar{R})$ and $S(\bar{R})$ (Here, $\bar{R}$ is $\bar{R}_{b, 1}$ or $\bar{R}_{b, 2}$ ) are same as shown above for each kind of (weak, medium-level or strong) liquid-pore wall interaction.

\section{RESULTS AND DISCUSSION}

\subsection{Value Of $R_{b, 2}$}

Figure 2 plots the values of $R_{b, 2}$ against $R_{b, 1}$ respectively for weak, medium-level and strong filtered liquid-pore wall interactions. For the weak and medium-level interactions, $R_{b, 2} \approx 1.4 R_{b, 1}$.The strong interaction considerably reduces the value of $\mathrm{R}_{\mathrm{b}, 2}$ when $R_{b, 1}$ is below $15 \mathrm{~nm}$. However, for $R_{b, 1}$ over $15 \mathrm{~nm}$, the interaction between the filtered liquid and the pore wall has a negligible influence on the value of $R_{b, 2}$.

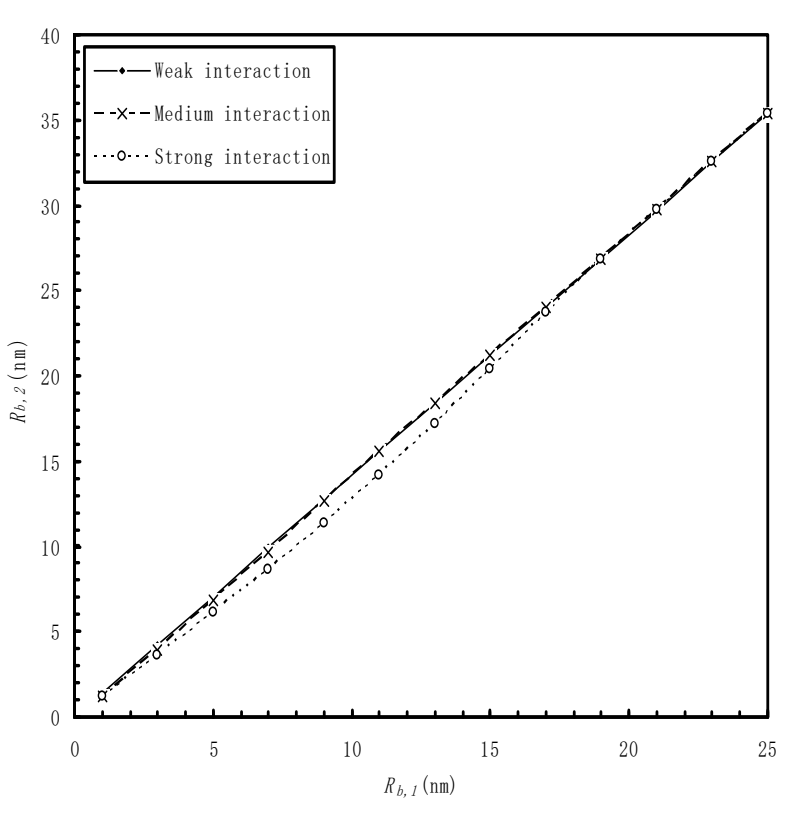

Fig. 2 Plot of the values of $R_{b, 2}$ against $R_{b, 1}$ respectively for weak, medium-level and strong filtered liquid-pore wall interactions.

\subsection{Values Of $F$ For Different Membrane Geometrical Parameter Values And Different Liquid-pore Wall Interactions}

Figures 3(a), (b) and (c) show that the value of $F$ i.e. the flow resistance of the membrane is significantly reduced with the increase of $\bar{R}_{b, 1}$ for whichever filtered liquid-pore wall interaction, especially when $\bar{R}_{b, 1}$ is small; This dependence appears most significant when the liquid-pore wall interaction is strong. For a given $\bar{R}_{b, 1}$, the increase of $\lambda_{1}$ i.e. the reduction of the membrane thickness $l$ very effectively reduces the value of $F$ i.e. the flow resistance of the membrane. These figures show that a larger value of $R_{b, 1}$ or/and a smaller thickness of the membrane both are very helpful for reducing the flow resistance of the membrane i.e. for improving the flux of the membrane.

\subsection{Values Of $F$ For Different Types Of Liquids}

Figures 4(a) and (b) respectively plot the values of $F$ against $R_{b, 1}$ for the three different liquids when $\lambda_{1}=1.0 E-04$ and $\lambda_{1}=1.0 E-03$. Liquid A, Liquid B and Liquid C respectively have weak, medium-level and strong interactions with the pore wall. When $R_{b, 1}$ is below $10 \mathrm{~nm}$, the liquid-pore wall interaction has a significant influence on the flow resistance of the membrane especially for low $R_{b, 1}$ values. When $R_{b, 1}$ is below $3 \mathrm{~nm}$, it is shown that the flow resistance of the membrane for Liquid $\mathrm{C}$ is over 100 or even over 1000 times that for Liquid A. Figures 4(a) and (b) show the applicability of the proposed membrane for a liquid-liquid separation if the mixed liquids have greatly different interactions with the pore wall. 


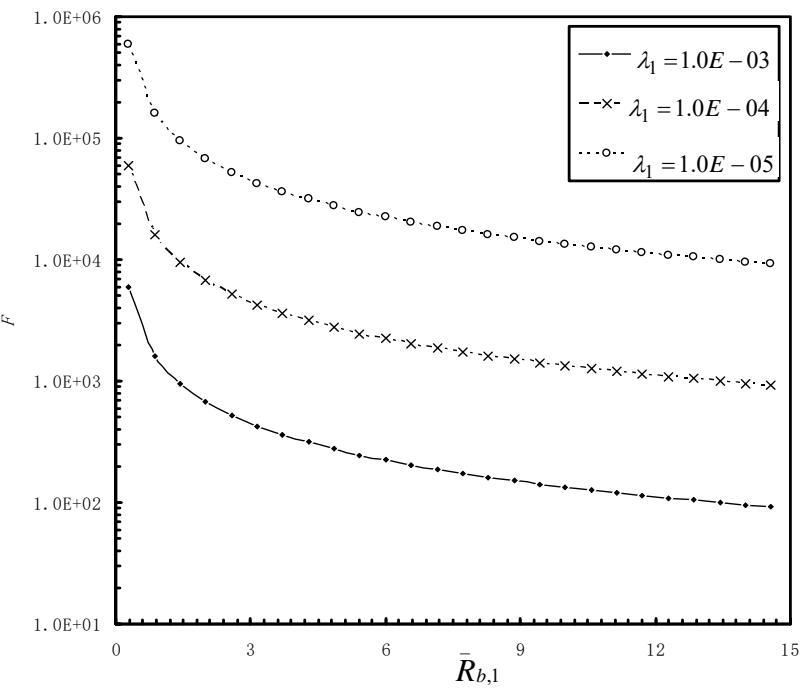

(a) For the weak liquid-pore wall interaction

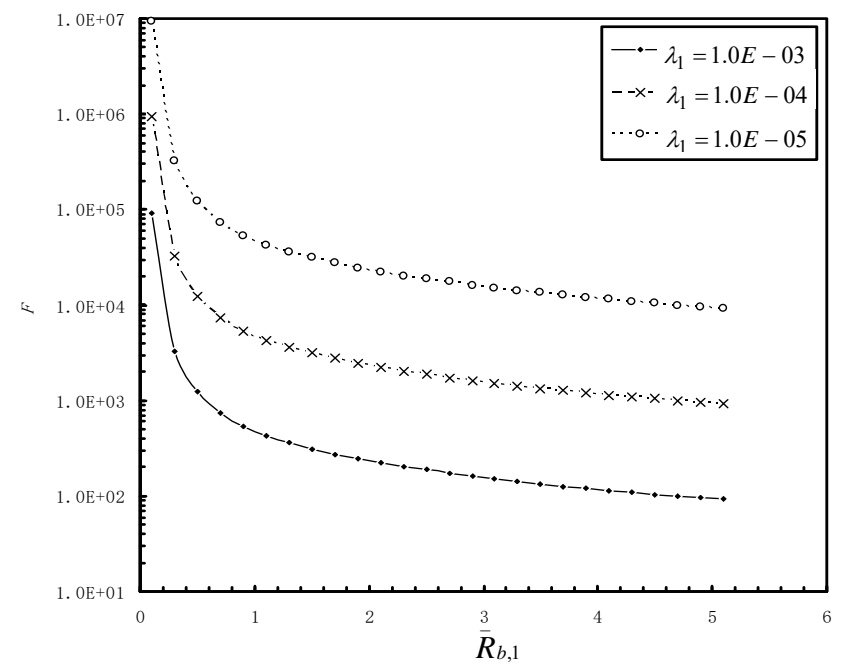

(b) For the medium-level liquid-pore wall interaction

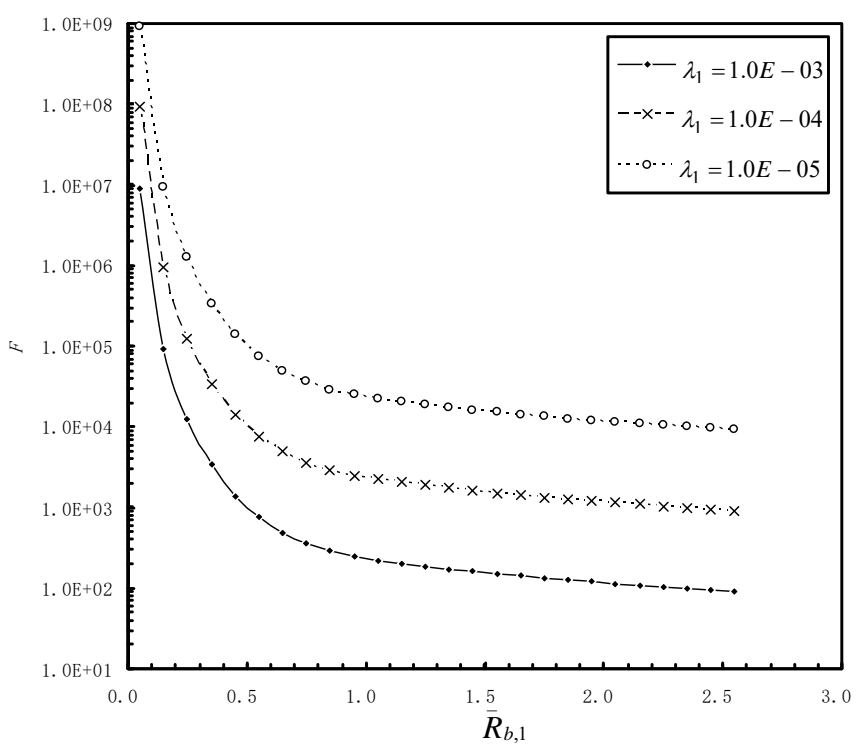

(c) For the strong liquid-pore wall interaction

Fig. 3 Plots of the values of $F$ against $\bar{R}_{b, 1}$ for different values of $\lambda_{1}$ and different filtered liquid-pore wall interactions, $\lambda_{1}=R_{b, 1} / l$.



(a) For $\lambda_{1}=1.0 E-04$

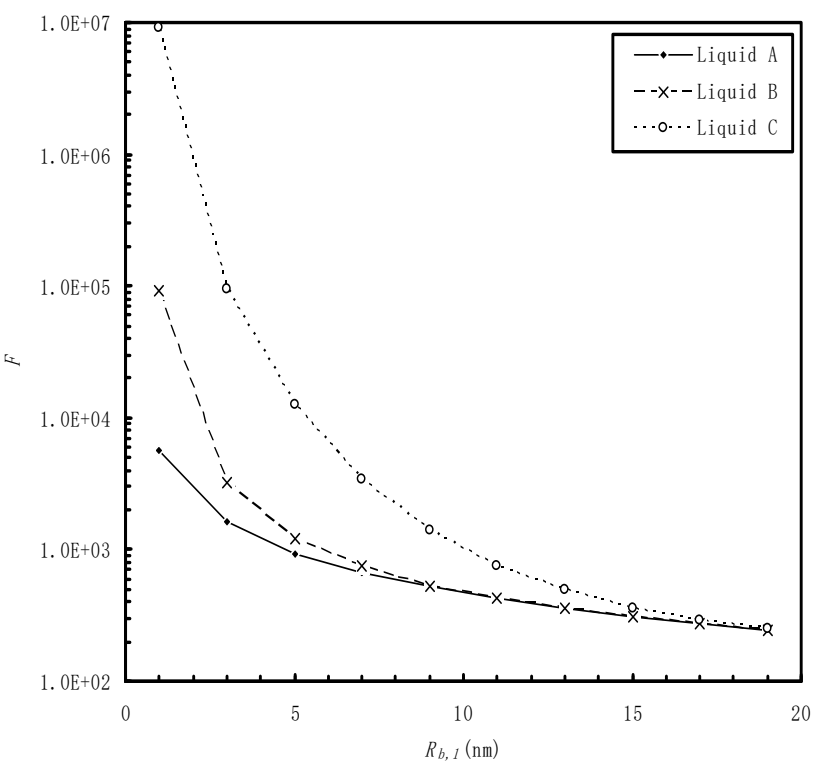

(b) For $\lambda_{1}=1.0 E-03$

Fig. 4 Plots of the values of $F$ against $R_{b, 1}$ for different types of liquids.

\section{CONCLUSIONS}

A tree-type cylindrical-shaped nanoporous filtering membrane is proposed. The flow resistance of this membrane is calculated for different operational parameter values and different filtered liquid-pore wall interactions, by using the flow factor approach model for a nanoscale flow. The design method for this membrane is obtained. It is obtained that the increase of the radius of the branch pore and/or the reduction of the membrane thickness both very effectively reduce the flow resistance of the membrane i.e. increase the flux of the membrane. For a small radius of the branch pore, the filtered liquid-pore wall interaction has a strong effect on the flow resistance of the membrane. This membrane can make the liquid with a weak interaction with the pore wall flow through while prevent the liquid with a strong interaction with the pore wall from coming through it, because of the greatly different flow resistances of the membrane for these liquids. This provides an indication that the proposed membrane can be used for a liquid-liquid separation. 


\section{REFERENCES}

Adiga, S. P., Jin, C., Curtiss, L. A., Monteiro-Riviere, N. A., and Narayan, R. J., 2009, "Nanoporous Membranes for Medical and Biological Applications,"Nanomedicine and Nanobiotechnology, 1, 568-581. https://doi.org/10.1002/wnan.50

Biffinger, J. C., Ray, R., Little, B., Ringeisen, B. R., 2007, “Diversifying Biological Fuel Cell Designs by Use of Nanoporous Filters,"Environmental Science \& Technology, 41, 1444-1449. https://doi.org/10.1021/es061634u

Fissel, W. H., Dubnisheva, A., Eldridge, A. N., Fleischman, A. J., Zydney, A. L., and Roy, S., 2009, "High-Performance Silicon Nanopore Hemofiltration Membranes," Journal of Membrane Science, 326, 58-63. https://doi.org/10.1016/j.memsci.2008.09.039

Li, N., Yu, S., Harrell, C., and Martin, C. R., 2004, "Conical Nanopore Membranes: Preparation and Transport Properties," Analytical Chemistry, 76, 2025-2030.

https://doi.org/10.1021/ac035402e

Surwade, S. P., Smirnov, S. N., Vlassiouk, I. V., Unocic, R. R., Veith, G. M., Dai, S., and Mahurin, S. M., 2015, "Water Desalination Using Nanoporous Single-Layer Grapheme," Nature Nanotechnology, 10, 459464.

https://doi.org/10.1038/nnano.2015.37

Venkatesan, B. M., Dorvel, B., Yemenicioglu, S., Watkins, N., Petrov, I., and Bshir, R., 2009, "Highly Sensitive, Mechanically Stable Nanopore Sensors for DNA Analysis," Advanced Materials, 21, 2771-2776. https://doi.org/10.1002/adma.200803786
Yang, S. Y., Ryu, I., Kim, H. Y., Kim, J. K., Jang, S. K., and Russell, T. P., 2006, "Nanoporous Membranes with Ultrahigh Selectivity and Flux for the Filtration of Viruses," Advanced Materials, 18, 709-712. https://doi.org/10.1002/adma.200501500

Zhang, Y. B., 2014, "Lubrication Analysis for a Line Contact Covering from Boundary Lubrication to Hydrodynamic Lubrication: Part I- Micro Contact Results," Journal of Computational and Theoretical Nanoscience, 11, 62-70. https://doi.org/10.1166/jctn.2014.3318

Zhang, Y. B., 2016, "The Flow Equation for a Nanoscale Fluid Flow," International Journal of Heat and Mass Transfer, 92, 1004-1008. https://doi.org/10.1016/j.ijheatmasstransfer.2015.09.008

Zhang, Y. B., 2017a, "Optimum Design for Cylindrical-Shaped Nanoporous Filtering Membrane,'International Communications in Heat and Mass Transfer, submitted.

Zhang, Y. B., 2017b, "Influence of Pore Wall Surface Property on Flux of Cylindrical-Shaped Nanoporous Filtering Membrane," Frontiers in Heat and Mass Transfer, 9, 26.

https://doi.org/10.5098/hmt.9.26

Zhang, Y. B., 2017c, "Transport in Nanotube Tree," International Journal of Heat and Mass Transfer, 114, 536-540. https://doi.org/10.1016/j.ijheatmasstransfer.2017.06.105

Zhang, Y. B., 2017d, "A Design Method for Nanofluidic Circuits," International Journal of Heat and Mass Transfer, submitted. 